\title{
Code Profiling in R: A Review of Existing Methods and an Introduction to Package GUIProfiler
}

\author{
by Angel Rubio and Fernando de Villar
}

\begin{abstract}
Code analysis tools are crucial to understand program behavior. Profile tools use the results of time measurements in the execution of a program to gain this understanding and thus help in the optimization of the code. In this paper, we review the different available packages to profile R code and show the advantages and disadvantages of each of them. In additon, we present GUIProfiler, a package that fulfills some unmet needs.

Package GUIProfiler generates an HTML report with the timing for each code line and the relationships between different functions. This package mimics the behavior of the MATLAB profiler. The HTML report includes information on the time spent on each of the lines of the profiled code (the slowest code is highlighted). If the package is used within the RStudio environment, the user can navigate across the bottlenecks in the code and open the editor to modify the lines of code where more time is spent. It is also possible to edit the code using Notepad++ (a free editor for Windows) by simply clicking on the corresponding line. The graphical user interface makes it easy to identify the specific lines which slow down the code.
\end{abstract}

The integration in RStudio and the generation of an HTML report makes GUIProfiler a very convenient tool to perform code optimization.

\section{Introduction}

Software profiling is the analysis of a computer program performed by measuring the time spent on each line of code, code coverage or memory usage during its execution. Profiling is the first step towards efficient programming. The development of efficient software depends on identification of key bottlenecks. Focusing the optimization only on the bottlenecks is known to maximize efficiency in both development time and program runtime (Wilson et al., 2014). In interpreted languages (including R) a few lines can form major bottlenecks. See for example Visser et al. (2015).

With the advent of data mining, data analytics and big data analysis, code profiling is gaining prominence. In these fields, one potential limitation to scientific advance is inefficient code. Since the factors that affect the execution time are difficult to foresee beforehand, and the bottlenecks (if the code is large) are especially difficult to identify, the need of a profiling tool is apparent. In addition to that, inefficient code is also prone to have bugs. Our experience is that profiling is also an indirect way to fix errors in software.

The base distribution of $\mathrm{R}$ includes a profiling tool that consists of the functions Rprof to start and stop the profiling and summaryRprof as a parser of the output. The description of Rprof given in the help file is: "Profiling works by writing out the call stack every interval seconds (...)". This means that $\mathrm{R}$ uses the operating system interrupts to sample and write the call-stack (by default every 20 msecs). Therefore, lines that take more than 20 msecs appear at least once in the file written by the internal $\mathrm{R}$ profiler. On the other hand, a "fast" line of code where the execution time is strictly less than 20 msecs may or may not appear in the output file (with the probability of being included proportional to its execution time). Therefore, the output of profiling the same code is not identical for different runs. Overall it can be noted that statistical profiling (i.e., the one implemented in R) intrudes very little on the executed code (i.e., it almost does not affect its execution time) and is considered to be an efficient way to achieve proper profiling.

In previous versions of $\mathrm{R}$ (prior to 3.0), Rprof only worked at the function level (i.e., the profiler only provided information on the functions in the stack). Since version 3.0, it is possible to perform line profiling. If the line.profiling option is selected, the file generated by Rprof also includes information on the specific line of code in the stack (not only the function). This extension made it possible to identify the specific lines that slow down the code.

The output of Rprof is quite simple: a file that shows the name and some other characteristics of a function every time it is found to be in the stack. Despite this simplicity, it is necessary to parse this file to understand the content and R provides the "summaryRprof function (...) that can be used to process the output file to produce a summary of the usage" (from its help file).

The functionality of summaryRprof falls short in some aspects. If a function is called several times, summaryRprof fails to identify the specific function call that is slowing down the computation. As 
functions can be nested in other functions, finding the true bottleneck is not obvious. In summaryRprof, it is not possible to track the hierarchy among the functions. summaryRprof was developed prior to the line.profiling option and does not take full advantage of this information. This fact will be shown in detail in the analysis of the different profile tools below.

These limitations have fostered the development of other command line functions and packages such as proftable, aprof, proftools, profr, and lineprof. proftable (Klevtsov, 2014) is a convenient command line function that parses the output of Rprof and shows the output in a reasonable and intuitive way. aprof (Visser et al., 2015) displays graphically the time spent on each line of code and provides an estimate on how optimizing a single line of code will affect the overall performance. It also shows the output of memory profiling. proftools (Tierney and Jarjour, 2013) includes useful command line tools to perform the profiling. Among other functionalities, it shows a graph of the different hierarchical relationships between the called functions. lineprof (Wickham, 2014a) can be considered an evolution of profr (Wickham, 2014b) and is developed by the same author. It is a profiling package that provides output integrated in the RStudio environment. It shows a nice graphical output and also includes memory profiling capabilities. In addition to these tools, the pbdPAPI package (Schmidt et al., 2015) offers access to low-level hardware counter information and is mainly used for advanced profiling. On the other hand, the most convenient package to test the performance of a single line of code is microbenchmark (Mersmann, 2014). It cannot be applied, though, to profile complex code. To our knowledge, these are all the available tools to aid in $\mathrm{R}$ profiling.

All these packages are, as most $\mathrm{R}$ packages, command line tools. Although they represent an important advance if compared with the summaryRprof function, none of them are especially userfriendly. In comparison, MATLAB ${ }^{\mathrm{TM}}$ (The MathWorks Inc., 2015) provides a profiler that includes a convenient user interface by means of an HTML report. Profiling MATLAB code is easy and straightforward with the aid of its profile tool. An $\mathrm{R}$ profiler with this convenient front-end that includes GUI capabilities would be highly desirable.

Fortunately, the development of this tool is not such a challenging task. Several packages such as Nozzle.R1 (Gehlenborg, 2013) or knitr (Xie, 2014) are available on CRAN that automatically generate HTML reports based on an easy syntax. GUIProfiler (de Villar and Rubio, 2015) is an R package that automatically generates an HTML report that summarizes the profiling results. These reports are generated with the aid of Nozzle.R1. Its integration in the RStudio environment makes it especially user friendly.

The following sections show how to use package GUIProfiler as well as provide a review on the profiling capabilities of the aforementioned tools using the same sample code for all of them.

\section{Case study using GUIProfiler}

The first lines of code are required for the installation of the package. As GUIProfiler is hosted on CRAN, the installation is straightforward:

install. packages ("GUIProfiler")

library("GUIProfiler")

If the package is properly installed, no errors should appear after calling the library command. GUIProfiler has a practical limitation that must be taken into account: The profiled code must be stored on an accessible file. It is therefore better to run a source command instead of writing the lines directly in the command line. The reason of this limitation is that the report is based on the output from Rprof and that it only includes information on the functions stored at files, not from the script that calls them. In addition to that, it only accepts one function per file. R does allow to include several functions in a single file. We are currently working to circumvent this limitation.

Here we present some sample code (included also in the GUIProfiler documentation) that will be used with all the profiling tools. The HTML report generated by package GUIProfiler is shown in Figure 1 and the code is,

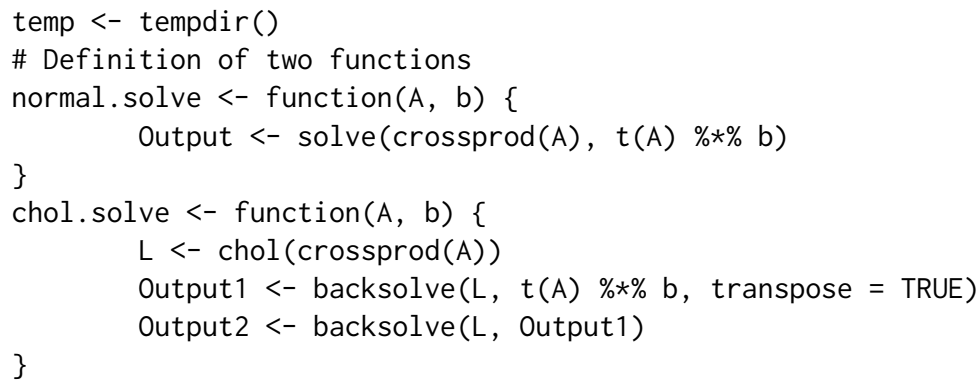




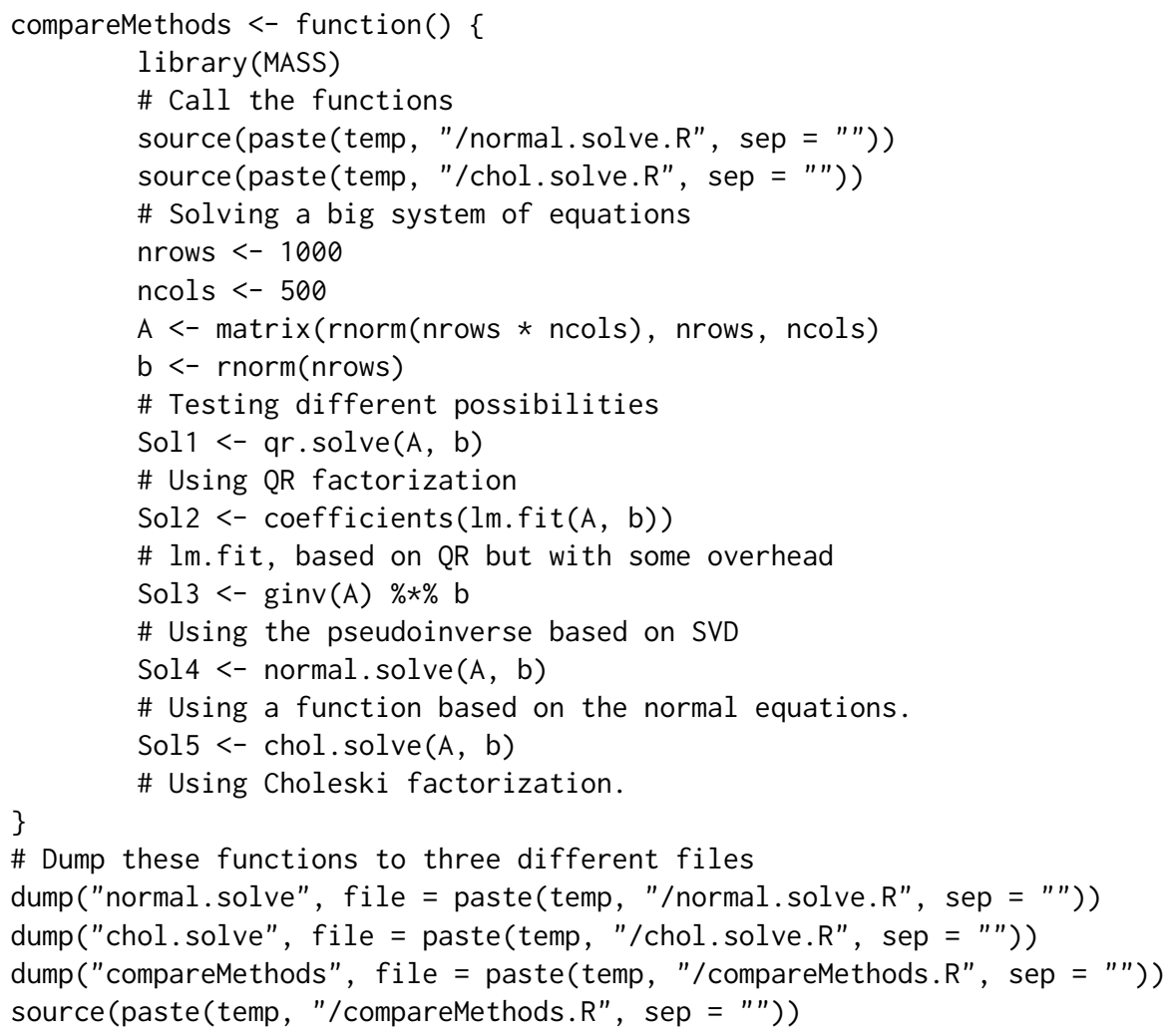

This code implements the minimum squares solution of a linear system of equations using different methods: QR factorization, the $1 \mathrm{~m}$. fit function (that internally also uses the QR factorization), computation of a generalized pseudoinverse (that internally uses the SVD factorization), using the normal equations and, finally, using the Choleski factorization.The solutions using either of them are identical, i.e., the vectors Sol1, Sol2, Sol3, Sol4 and Sol5 are identical up to computer precision.

The lines to profile the code are only the last ones in the example code of the RRprofReport function. Specifically,

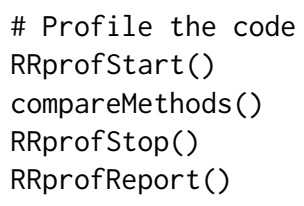

Each of these lines are self-explanatory. In the first line we activate and start the GUIProfiler. The following line executes the function that is being profiled. Once this line finishes, the profiling is stopped and the last line generates the report based on the output of the R profiler. In the RStudio environment, this report is shown in the viewer pane. In addition, the markers pane indicates the lines of code where more time was spent. It is possible to navigate through the source code by simply clicking on the corresponding markers.

If the program is not executed in the RStudio environment, RRprofReport() opens a new browser window. Figure 1 shows a snapshot of the generated report. The report consists of two groups of tables: a summary of the called functions with the time spent on each of them and a group of tables with the time spent on each line of code for each function. A convenient feature, if the browser is the Internet Explorer and Notepad++ is installed, is that the line numbers of the functions are clickable: Once a line number is clicked, the corresponding file is opened with the cursor on the selected line (as shown in Figure 2). On the right panel of Figure 2, the layout of GUIProfiler is shown in the RStudio environment. Note that RStudio version $\geq 0.99$ is required. We tested GUIProfiler on RStudio 0.99.467. The navigation across the different functions can be done using the markers tab.

\section{Comparison between different profiling tools}

Using the same example, we compare the functionalities of the different profiling tools in their ability to provide insight on the profiled code. All the profiling tools (including GUIProfiler) manipulate the file generated by Rprof to provide a more readable and useful output. Therefore, most of the code to profile a function is shared by the different packages: First of all there is a call to Rprof to start 


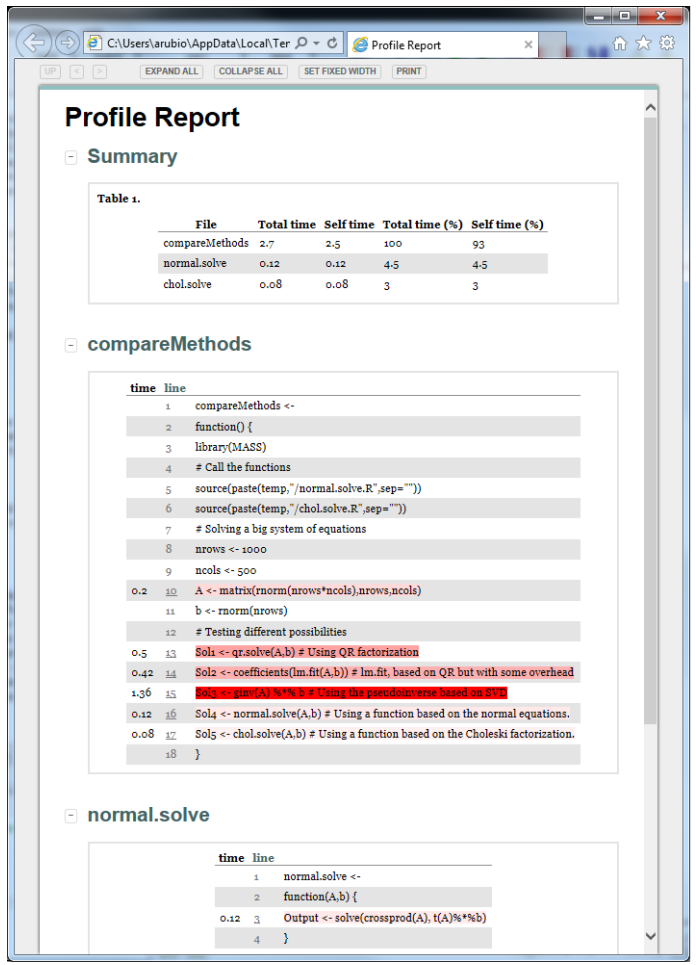

Figure 1: HTML report generated by GUIProfiler.

\begin{tabular}{ccccccc}
\hline & $\begin{array}{c}\text { HTML } \\
\text { report }\end{array}$ & $\begin{array}{c}\text { Graphical } \\
\text { output }\end{array}$ & $\begin{array}{c}\text { Function } \\
\text { nesting }\end{array}$ & $\begin{array}{c}\text { Line } \\
\text { profiling }\end{array}$ & $\begin{array}{c}\text { Memory } \\
\text { profiling }\end{array}$ & $\begin{array}{c}\text { Connection } \\
\text { with editors }\end{array}$ \\
\hline GUIProfiler & $\checkmark$ & $\checkmark$ & $\checkmark$ & $\checkmark$ & $x$ & $\checkmark\left(^{*}\right)$ \\
summaryRprof & $x$ & $x$ & $x$ & $\checkmark$ & minimal & $x$ \\
proftable & $x$ & $x$ & $\checkmark$ & $\checkmark$ & $x$ & $x$ \\
aprof & $x$ & $\checkmark$ & $\checkmark$ & $\checkmark$ & $\checkmark$ & $x$ \\
proftools & $x$ & $\checkmark$ & $\checkmark$ & $x$ & $x$ & $x$ \\
profr & $x$ & $\checkmark$ & $\checkmark$ & $x$ & $x$ & $x$ \\
lineprof & $\checkmark$ & $\checkmark$ & $\checkmark$ & $\checkmark$ & $\checkmark$ & $x$ \\
\hline MATLAB & $\checkmark$ & $\checkmark$ & $\checkmark$ & $\checkmark$ & $\checkmark\left(^{* *}\right)$ & $\checkmark$ \\
\hline
\end{tabular}

Table 1: Comparison of different profile tools for R. ${ }^{*}$ ) GUIProfiler connects the results with RStudio and with the Notepad++ editor. $\left.{ }^{* *}\right)$ Non-documented characteristic.

profiling, the code itself to be profiled and a second call to Rprof to stop profiling. The differences between them are the way the output of Rprof is summarized and displayed.

Table 1 shows a comparison between the functionalities of Rprof parsers used to profile $\mathrm{R}$ code. The first column indicates if the package generates an HTML report. The second column indicates if the package generates some visual graphical output to show the results. The "Function nesting" column shows whether the package is able to display the hierarchy across the function calls. The "Line profiling" column states whether the package provides information related with each of the lines in the code, not only the functions (i.e., whether it takes advantage of the line.profiling option). The "Memory profiling" column states whether the package shows results of profiling memory usage and finally, the "Connection with editors" column states whether the package has a direct link with an editor to fix the potential bottlenecks.

We also included the features of the MATLAB profiler. As can be seen, it offers all these functionalities. One anecdotal note of the MATLAB profiler is that, even though it implements memory profiling (in a very effective and user friendly manner), this feature is non-documented. summaryRprof, lineprof and aprof implement memory profiling in R. lineprof and GUIProfiler provide an HTML report. GUIProfiler is the only one that provides a connection with editors.

We include Table 2 to show the dependencies for each of the packages. As a general rule, packages with few dependencies are easier to install and to run in different conditions (i.e. on a server 

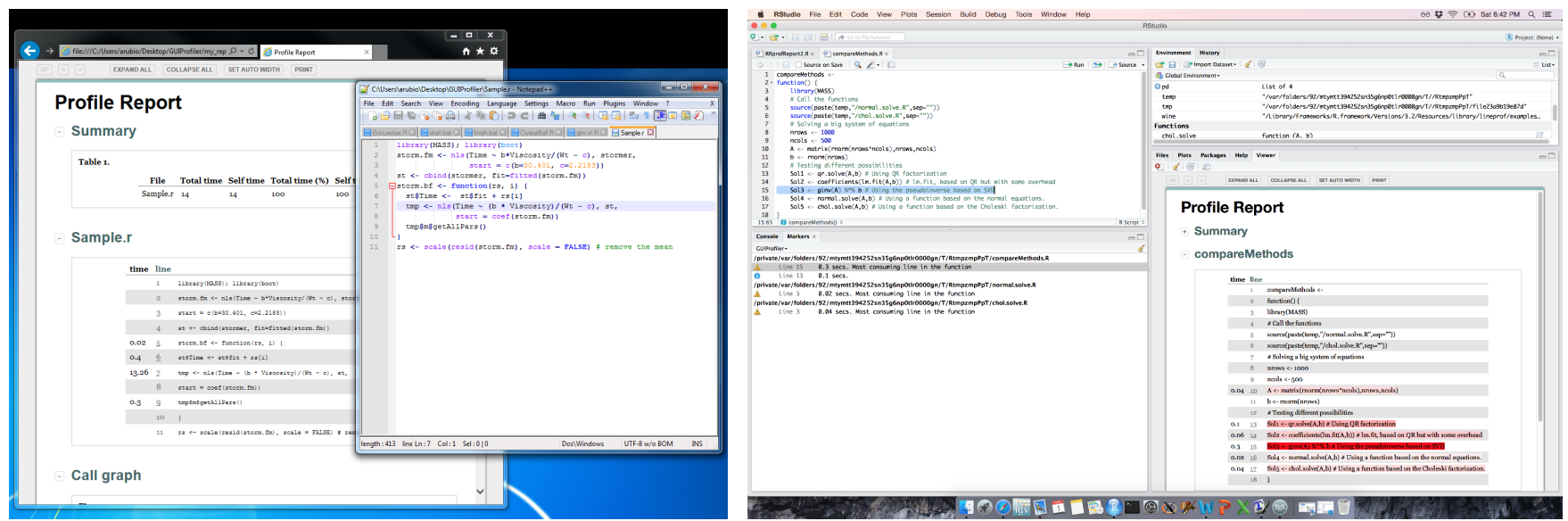

Figure 2: Layout of GUIProfiler. Left: Internet Explorer and Notepad++. Right: RStudio environment. The report is shown in the viewer tab on the right, and the markers tab on the left can be used to navigate across the code.

\begin{tabular}{ll}
\hline Tool & Dependencies \\
\hline GUIProfiler & $\begin{array}{l}\text { Nozzle.R1, Rgraphviz (Hansen et al., 2015), } \\
\text { graph (Gentleman et al., 2015), proftools }\end{array}$ \\
summaryRprof & None \\
proftable & None \\
aprof & grDevices \\
proftools & Rgraphviz, graph \\
profr & stringr (Wickham, 2015), plyr (Wickham, 2011) \\
lineprof & devtools (installation; Wickham and Chang, 2015), \\
& environment (C compiler), \\
& shiny (Chang et al., 2015, and its dependencies) \\
\hline
\end{tabular}

Table 2: Dependencies of the packages analyzed. Only packages not included in the standard distribution are mentioned.

enviroment). The following sections describe how to profile the example using the tools shown in Table 1.

\section{summaryRprof}

The code to perform the profiling of the example using summaryRprof is:

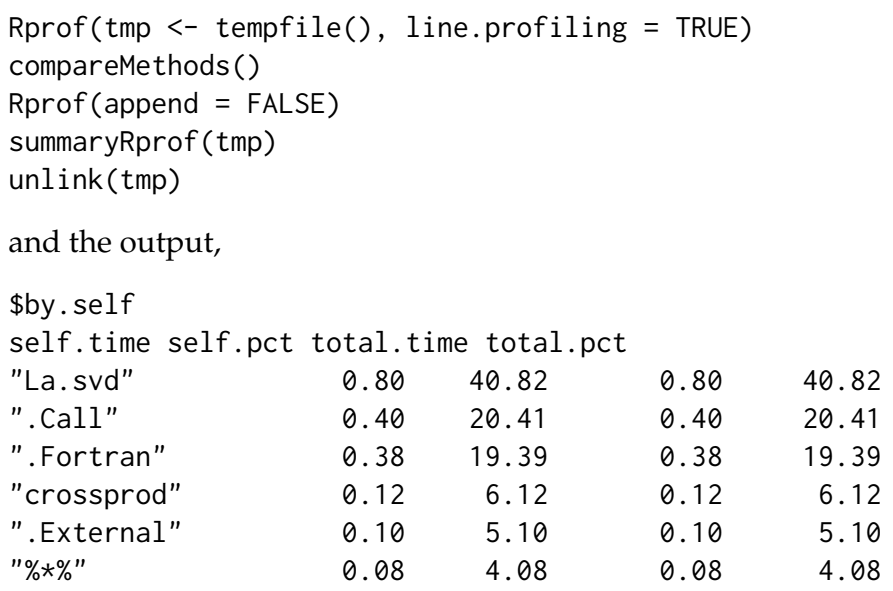

...More lines not included...

\$by. total 


\begin{tabular}{|c|c|c|c|c|}
\hline "compareMethods" & 1.96 & 100.00 & 0.00 & 0.00 \\
\hline "ginv" & 0.90 & 45.92 & 0.00 & 0.00 \\
\hline "svd" & 0.82 & 41.84 & 0.02 & 1.02 \\
\hline "La.svd" & 0.80 & 40.82 & 0.80 & 40.82 \\
\hline ".Call" & 0.40 & 20.41 & 0.40 & 20.41 \\
\hline "coefficients" & 0.40 & 20.41 & 0.00 & 0.00 \\
\hline "lm.fit" & 0.40 & 20.41 & 0.00 & 0.00 \\
\hline ".Fortran" & 0.38 & 19.39 & 0.38 & 19.39 \\
\hline "qr" & 0.38 & 19.39 & 0.00 & 0.00 \\
\hline
\end{tabular}

...More lines not included...

\$sample. interval

[1] 0.02

\$sampling. time

[1] 1.96

If we focus on the "by. self" part, the information is not too useful: the .Call or . Fortran functions can be used anywhere and depending on their argument, their behavior is completely different. On the other hand, the crossprod function is used many times in the code and the table only shows the overall time spent on it. Although the line.profiling option was set, line information does not appear anywhere in the output. The latest version of $\mathrm{R}$ (by setting summaryRprof ( $\mathrm{tmp}$, lines = "show")) provides basic information on line profiling.

The "by. total" part states that the most costly functions are ginv, svd and La.svd. However, these functions are in fact all the same: ginv calls svd that, in turn, calls La.svd. The output does not show this hierarchy in the calls to the function. In addition, the summaryRprof output does not show locations of the calls to the corresponding functions within the code. The output presents therefore serious limitations for its practical use that can be solved with other tools described below.

proftable

proftable is a convenient function that solves some of the aforementioned problems of summaryRprof: Each line of code is clearly identified and can be easily tracked. The function can be accessed from GitHub. The code to run the example is:

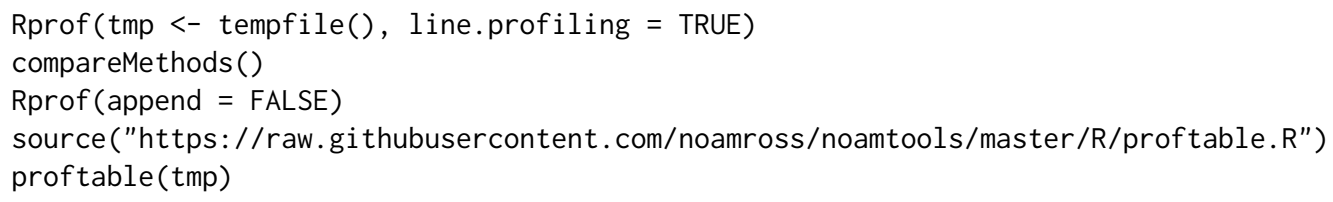

And the output,

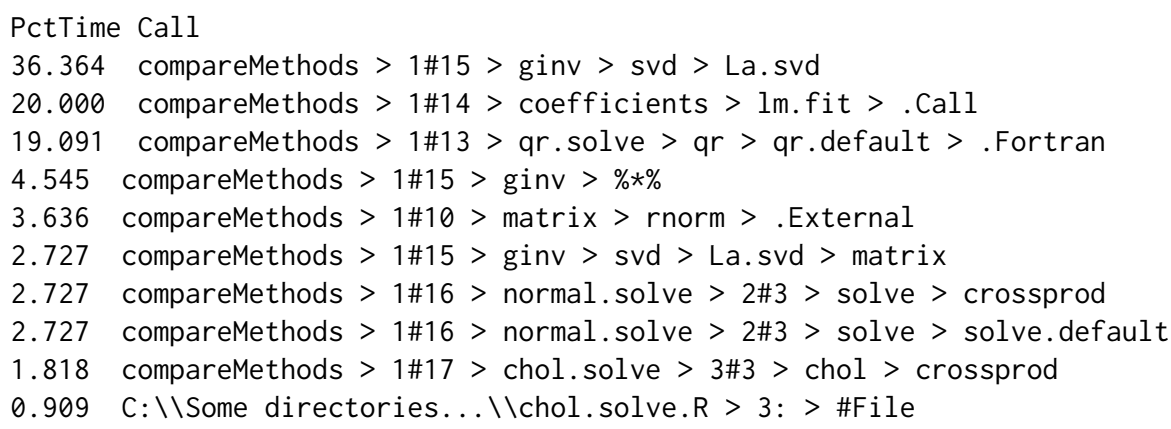

\#File 1: C: \Users\arubio\AppData\Local\Temp\RtmpEH6kiO/compareMethods.R

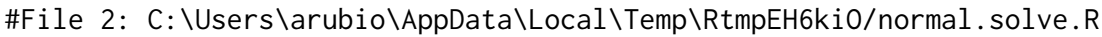
\#File 3: C:\Users\arubio\AppData\Local\Temp\RtmpEH6kiO/chol.solve.R

Parent Call: None

Total Time: 2.2 seconds

Percent of run time represented: $94.5 \%$ 

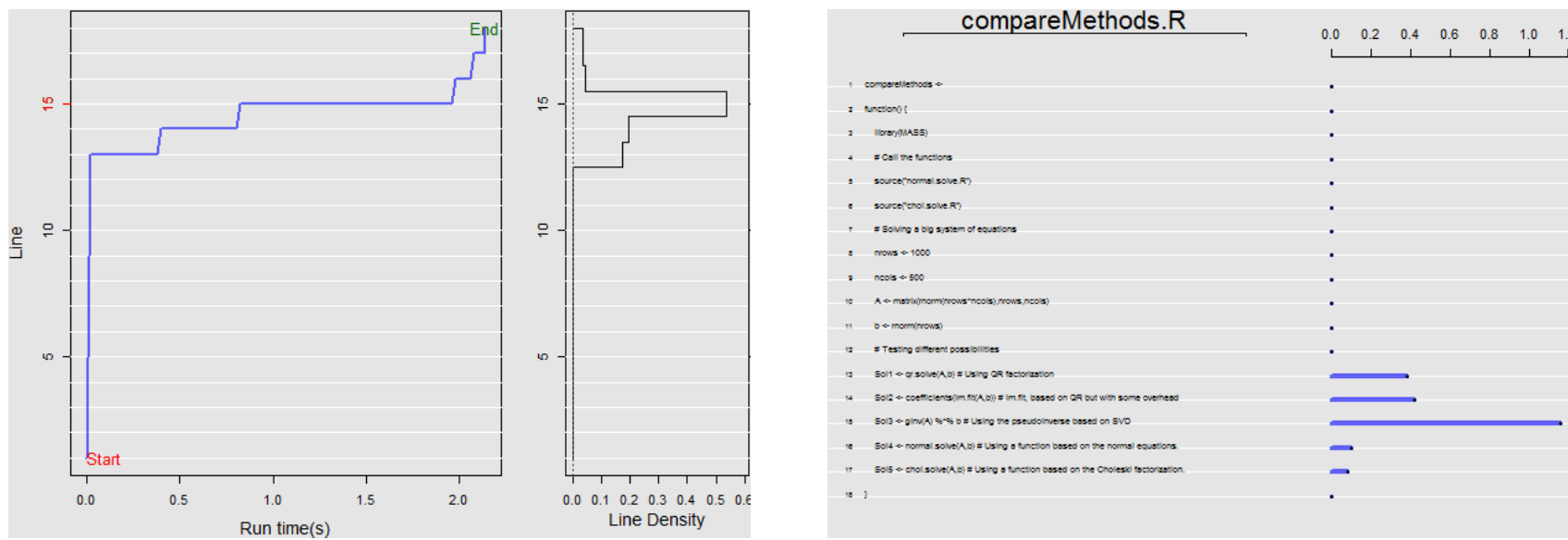

Figure 3: Output generated by profileplot of an 'aprof' object. Left panel: accumulated time and time spent on each line of code. Right: content for each line of code and the spent on each of them.

In this case the hierarchical relationships between ginv, svd and La.svd (as well as other ones) are clearly stated. Each line of code may appear several times. This "excess" of information can be useful to identify within each line of code which is the most costly part. However, it is also confusing. For example, line 15 of the first archive appears several times in the list since svd and matrix multiplication (both inside the ginv function) are costly operations.

proftable includes the location of each line in each file, making their analysis within a context easier. proftable is a simple, yet very useful tool.

\section{aprof}

aprof also works with the output of Rprof and provides visual aids based on line profiling. It helps to identify the most promising sections of code to optimize. One interesting unique functionality is that aprof also projects the potential gains. The last version has also memory profiling included. The code to run aprof is:

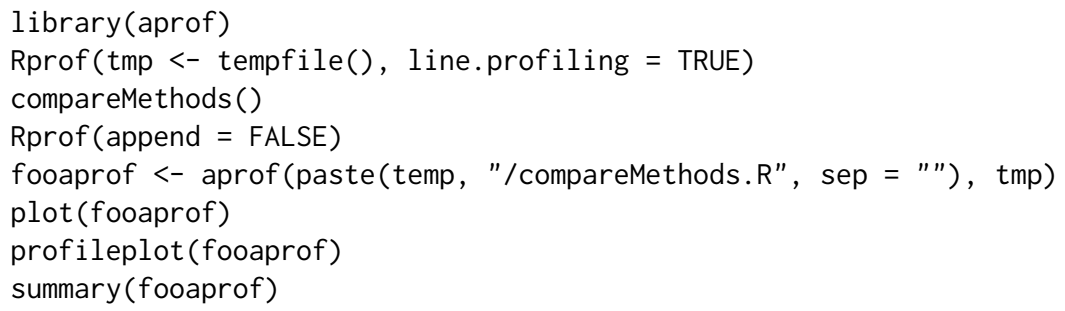

Running the provided code in aprof generates nice plots that describe the time spent on each line of code (and the code itself). Figure 3 shows the aprof output. The summary function estimates the speed-up by optimizing a single line of code (or all the lines of code). The output to the console is:

Largest attainable speed-up factor for the entire program

when 1 line is sped-up with factor (S):

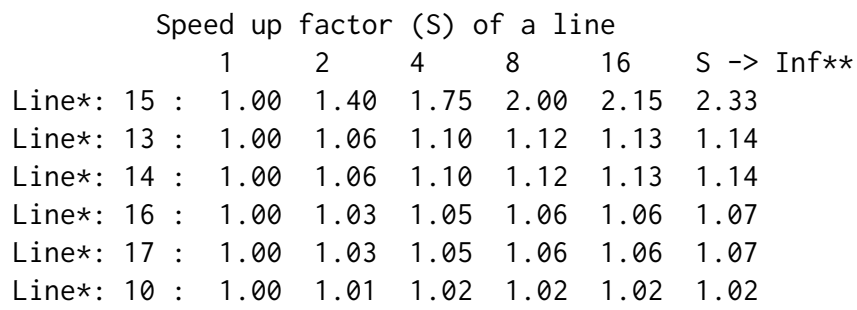

Lowest attainable execution time for the entire program when 


\begin{tabular}{lllllll}
\multicolumn{7}{c}{ Speed up factor $(\mathrm{S})$ of a line } \\
All lines & 2.840 & 1.420 & 0.710 & 0.355 & 0.178 \\
Line*: $15:$ & 2.840 & 2.030 & 1.625 & 1.423 & 1.321 \\
Line*: $13:$ & 2.840 & 2.670 & 2.585 & 2.543 & 2.521 \\
Line*: $14:$ & 2.840 & 2.670 & 2.585 & 2.543 & 2.521 \\
Line*: $16:$ & 2.840 & 2.750 & 2.705 & 2.683 & 2.671 \\
Line*: $17:$ & 2.840 & 2.750 & 2.705 & 2.683 & 2.671 \\
Line*: $10:$ & 2.840 & 2.810 & 2.795 & 2.788 & 2.784 \\
Total sampling time: & 2.84 seconds \\
* Expected improvement at current scaling \\
** Asymtotic max. improvement at current scaling
\end{tabular}

The line of code 'fooaprof <-aprof ("myfile.R..."' can be run for each of the files to get the profiling of all the executed functions. In this case it can be done by,

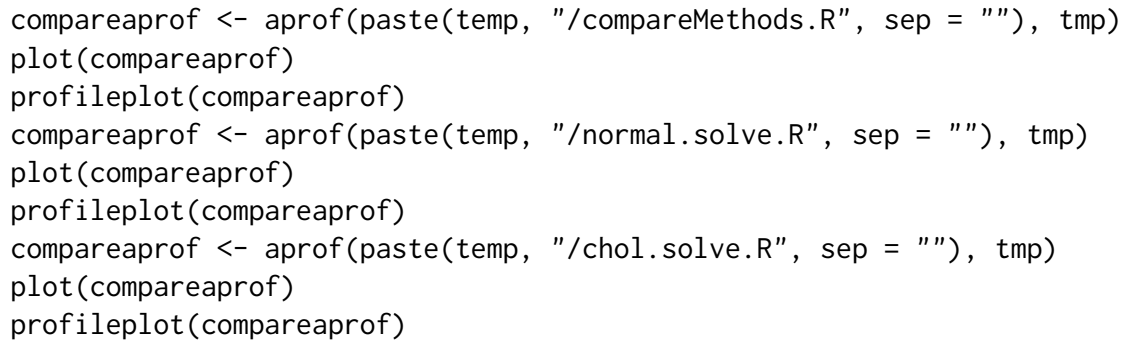

Alternatively, if the user wants to go into more detail for each of the functions, targetedSummary can be used to disentangle nested functions.

The information provided by aprof and GUIProfiler is very similar: aprof provides the output as an image and GUIProfiler as an HTML report.

\section{proftools}

proftools provides tools for examining Rprof profile output. It shows graphically the dependencies among the different functions and the time spent on each of them. The code to profile the example is:

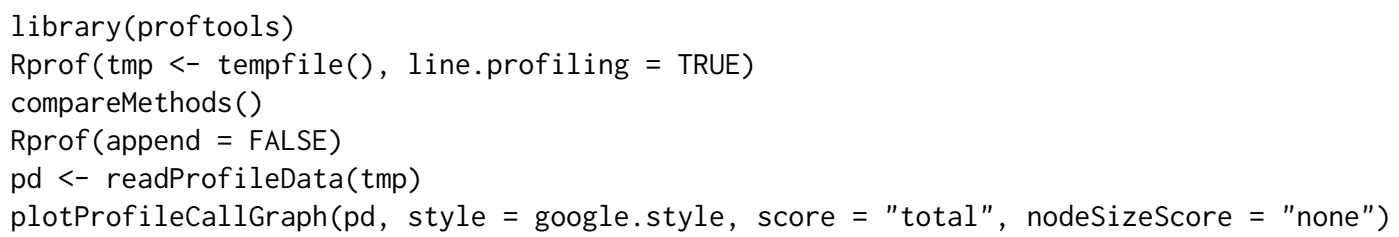

The user can get this information in the console by using the function printProfileCallGraph. The corresponding output is for this example:

\begin{tabular}{|c|c|c|c|c|}
\hline index & $\%$ time & $\%$ self & $\%$ children & name \\
\hline$[1]$ & 100.00 & 0.00 & 100.00 & compareMethods \\
\hline 1.06 & 0.00 & \multicolumn{2}{|c|}{$\% \star \%[15]$} & \\
\hline 0.00 & 2.13 & \multicolumn{2}{|c|}{ chol.solve [19] } & \\
\hline 0.00 & 21.28 & \multicolumn{2}{|c|}{ coefficients [8] } & \\
\hline 0.00 & 43.62 & \multicolumn{2}{|c|}{ ginv [2] } & \\
\hline 0.00 & 6.38 & \multicolumn{2}{|c|}{ matrix [13] } & \\
\hline 0.00 & 3.19 & \multicolumn{2}{|c|}{ normal.solve [16] } & \\
\hline 0.00 & 22.34 & \multicolumn{2}{|c|}{ qr.solve $[6]$} & \\
\hline & ----- & -- & -- & ------ \\
\hline 0.00 & 43.62 & \multicolumn{2}{|c|}{ compareMethods $[1]$} & \\
\hline [2] & 43.62 & 0.00 & 43.62 & ginv [2] \\
\hline 4.26 & 0.00 & \multicolumn{2}{|c|}{$\% * \% \quad[15]$} & \\
\hline 0.00 & 39.36 & \multicolumn{2}{|c|}{ svd $[4]$} & \\
\hline \multicolumn{5}{|c|}{ 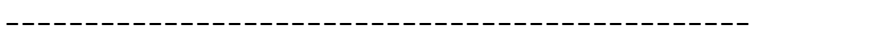 } \\
\hline 39.36 & 0.00 & \multicolumn{2}{|c|}{ svd $[4]$} & \\
\hline [3] & 39.36 & 39.36 & 0.00 & La.svd [3] \\
\hline
\end{tabular}




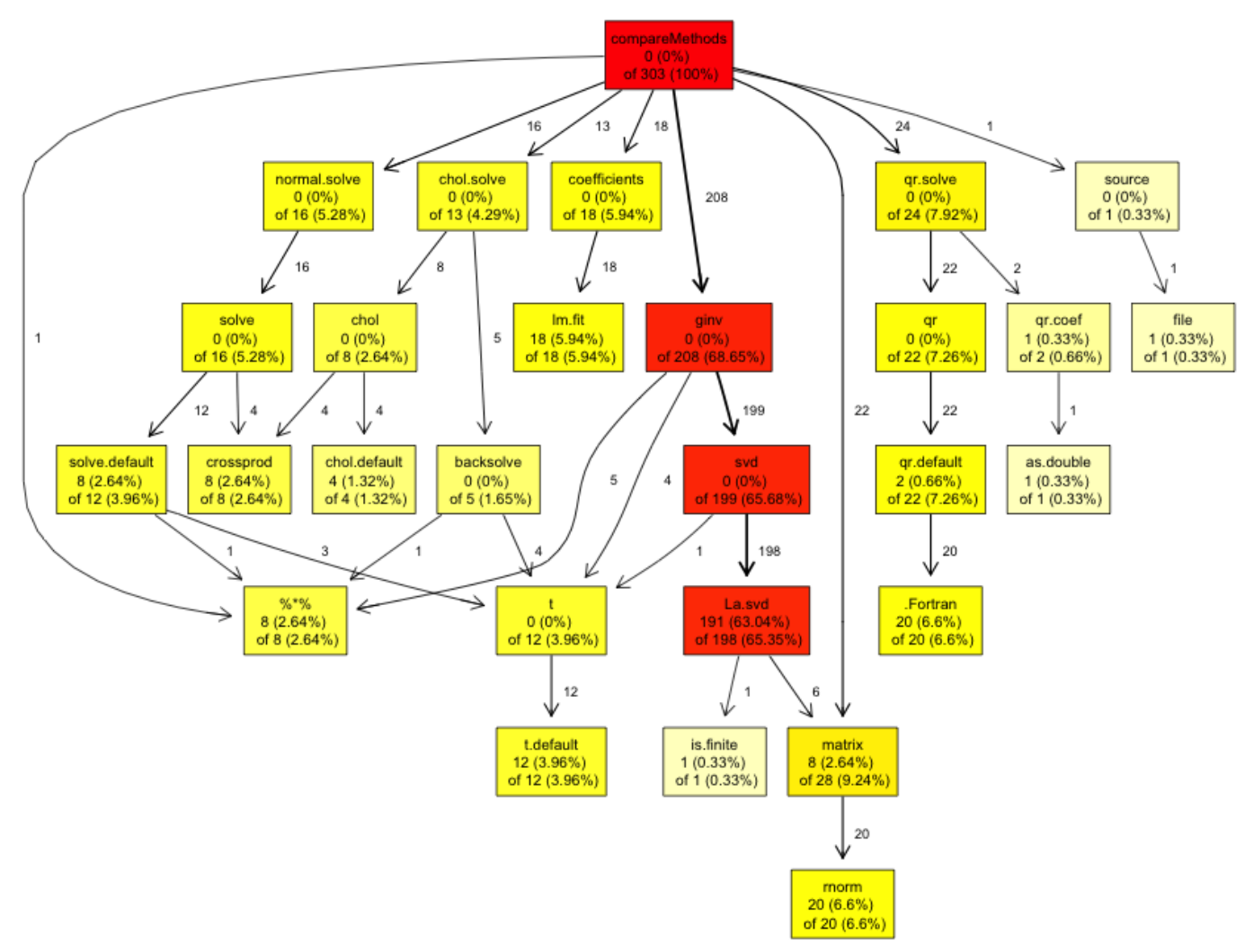

Figure 4: Output of proftools.

\begin{tabular}{|c|c|c|c|}
\hline 0.00 & 39.36 & ginv [2] & \\
\hline [4] & 39.36 & 39.36 & svd [4] \\
\hline 39.36 & 0.00 & La.svd [3] & \\
\hline 21.28 & 0.00 & qr.default $[11]$ & \\
\hline 1.06 & 0.00 & qr.coef [23] & \\
\hline [5] & 22.34 & 22.34 & .Fortran [5] \\
\hline 0.00 & 22.34 & compareMethods & \\
\hline [6] & 22.34 & $0.00 \quad 22.34$ & qr.solve [6] \\
\hline 0.00 & 21.28 & $\operatorname{qr}[10]$ & \\
\hline 0.00 & 1.06 & qr.coef [23] & \\
\hline 21.28 & 0.00 & lm.fit [9] & \\
\hline$[7]$ & 21.28 & $\begin{array}{ll}21.28 & 0.00\end{array}$ & .Call [7] \\
\hline
\end{tabular}

... Additional lines not shown here...

Figure 4 shows the dependencies between the different functions. Probably, this tool provides the most intuitive representation of the different relationships among the functions in the code. We have taken advantage of this in GUIProfiler and this graph is also included in the generated report.

proftools is, however, "function based" and the line.profiling option is not used at all. In fact, if Rprof is used without the line.profiling option, the result is identical.

\section{profr}

profr is one of the oldest packages to parse the Rprof output. The first version appeared in May, 2008. Its usage, as for the other tools described here, is straightforward:

library (profr)

profcompareMethods <- $\operatorname{profr}(\operatorname{compareMethods}())$ 


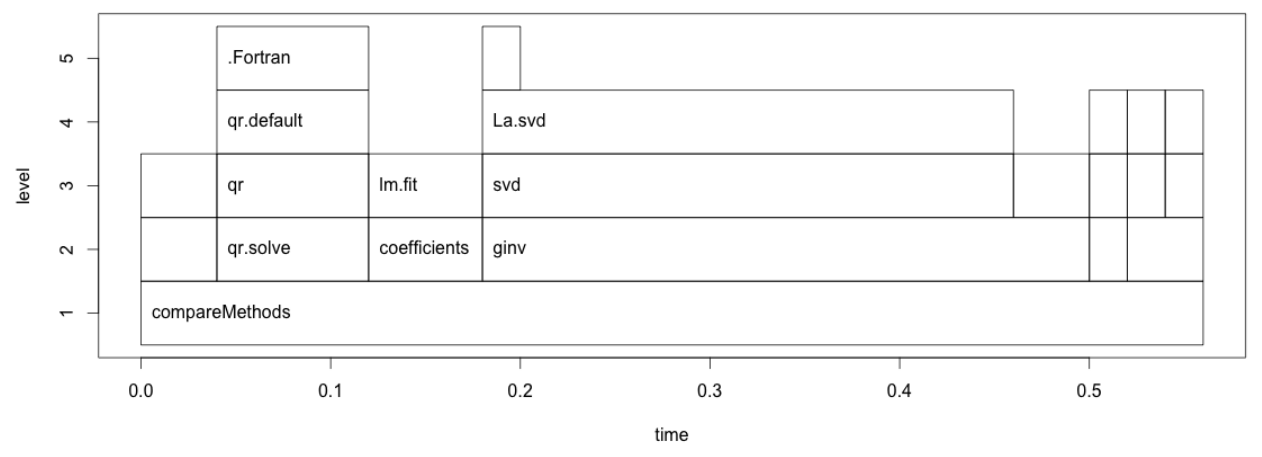

Figure 5: Output of profr.

head (profcompareMethods)

plot (profcompareMethods)

The output provided by profr is the following:

\begin{tabular}{|c|c|c|c|c|c|c|c|c|c|c|}
\hline & & g_id & $t_{-}$id & $f$ & start & end & $\mathrm{n}$ & leaf & time & source \\
\hline 8 & 1 & 1 & 1 & compareMethods & 0.00 & 0.76 & 1 & FALSE & 0.76 & .GlobalEnv \\
\hline 9 & 2 & 1 & 1 & matrix & 0.00 & 0.08 & 1 & FALSE & 0.08 & base \\
\hline 10 & 2 & 2 & 1 & qr.solve & 0.08 & 0.20 & 1 & FALSE & 0.12 & base \\
\hline 11 & 2 & 3 & 1 & coefficients & 0.20 & 0.26 & 1 & FALSE & 0.06 & stat \\
\hline 12 & 2 & 4 & 1 & ginv & 0.26 & 0.66 & 1 & FALSE & 0.40 & MAS \\
\hline 13 & 2 & 5 & 1 & normal. solve & 0.66 & 0.74 & 1 & FALSE & 0.08 & balEr \\
\hline
\end{tabular}

There is a useful plot command that shows the time spent on each function. In turn, the functions are grouped by levels. The output is shown in Figure 5.

profr was (with summaryRprof) the first attempt to display the result of profiling $\mathrm{R}$ code. It was developed before line.profiling was available and does not make use of it. The graphical representation is much more informative than the text output. However, although the hierarchical tree of proftools and the graph shown in profr show basically the same information, in our opinion, the tree is more visually apparent and attracts attention directly to the bottlenecks of the code.

\section{lineprof}

lineprof can be considered as an evolution of profr (both have the same developer and maintainer). Although it is not yet on CRAN, the installation from GitHub is straightforward.

install. packages ("devtools")

library (devtools)

devtools: : install_github("hadley/lineprof")

lineprof presents some characteristics that make it unique: It is integrated in the RStudio environment using the shiny package, it provides memory profiling out of the box and finally, using the shiny environment it is possible to navigate across the different functions to find out the bottlenecks in the code. The application to the example is also straightforward:

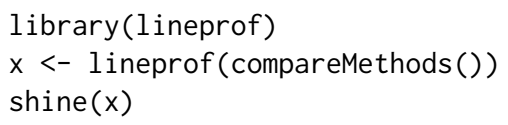

The resulting figure shows the output in the viewer pane in RStudio. The code for each function is shown there. The blue lines of code are hyperlinks to the corresponding functions. The last column represents the memory spent on each line of code. It can be seen that the creation of the $1000 \times 500$ matrix is the most expensive line of code in terms of memory use.

We experienced some minor issues when using lineprof that are worth mentioning. The representation in RStudio is a little bit buggy: The columns for each of the results (time spent, memory used, etc) are not properly shown. The navigation across the functions, although very intuitive, does not link directly to the RStudio editor to work on the code. Finally, when working on the shiny environment, the console appears to be busy and the user has to break it using Ctrl-C or Esc. We expect that most of these minor problems will be fixed in the stable release. 


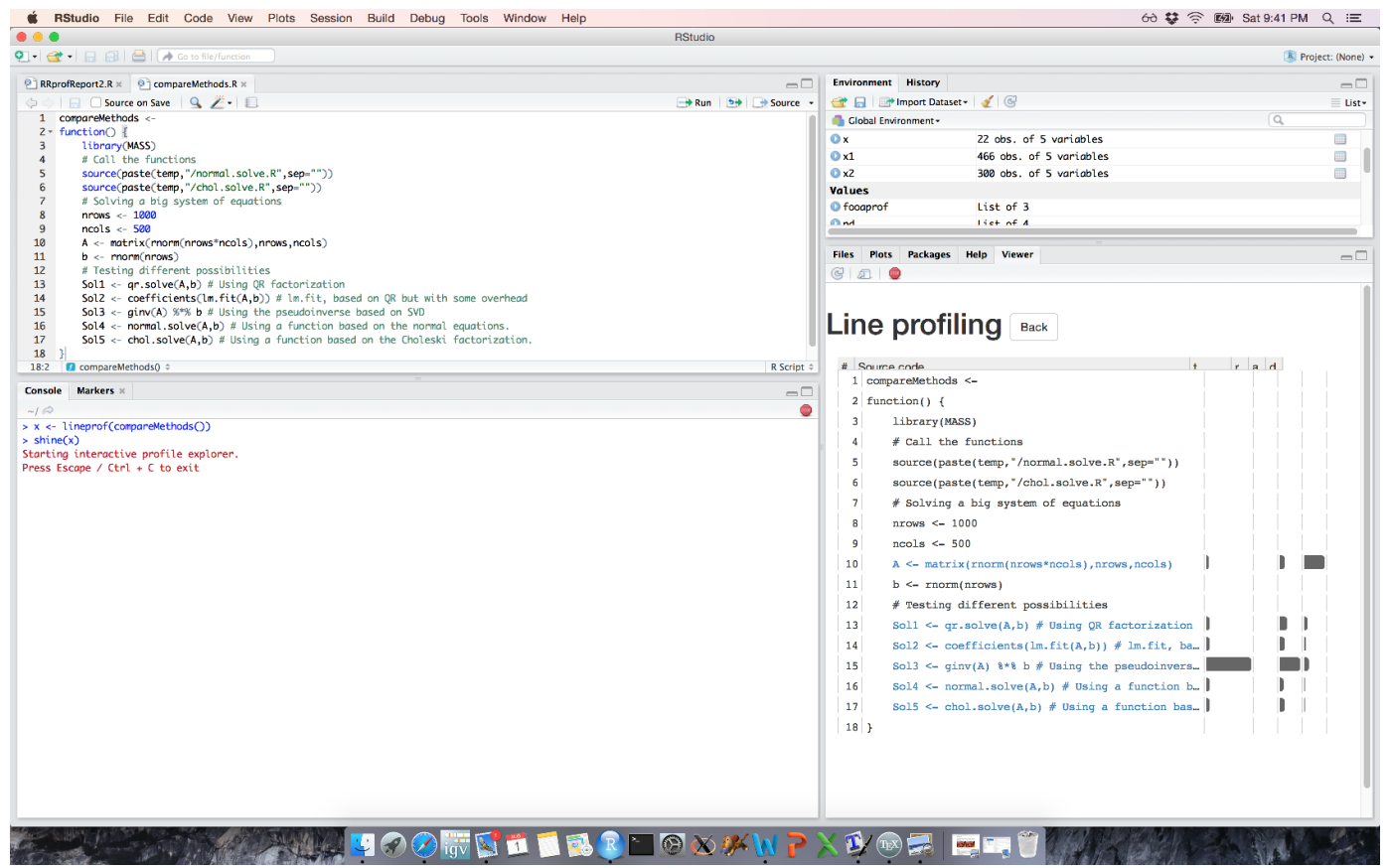

Figure 6: Output of lineprof.

\section{Discussion and conclusion}

This paper describes GUIProfiler, a graphical user interface to the line profiler provided by $\mathrm{R}$ and reviews, in relation to GUIProfiler, all other available profiling packages. Based on an example, we presented how to profile this code using the different packages and compared their advantages and disadvantages.

This review shows that, in spite of being far from the intuitiveness and user friendliness of the MATLAB profiler, the different developed tools are getting closer to it. For example, the graphical display of the relationship between functions in proftools is useful and intuitive and MATLAB does not provide anything like it. lineprof allows the navigation across the different functions very much like MATLAB's profiler does.

Regarding the described tools, proftable, profr and proftools are comparable. proftable provides an abstract on the time spent on each line of the code that is clearer and more useful than the one from summaryRprof. profr and proftools show graphically the relationships between the different profiled functions and the time spent on each of them. Unfortunately, neither of them provide profiling at the line level.

The other group is formed by aprof, lineprof and GUIProfiler. All of them provide similar information: time spent on each line of code stating the actual code of the line. There are also some differences among them. aprof shows these results using plots and provides estimates of the expected improvements when speeding up the most costly lines of code. lineprof displays these results using the shiny environment. Both lineprof and aprof provide memory profiling. GUIProfiler, on the other hand, builds an HTML report which is interactively linked with the program code. The preference between both tools may be a question of taste, but in our opinion, the HTML report is more advantageous.

We would like to note that there might be other characteristics which could be taken into account when evaluating a profiling tool such as those considered in this paper. For example, we assumed that graphical output is something desirable. However, this is not the case if the profiled code is run on a server with no graphical capabilities. In the case of lineprof, for example, a runtime environment is required to compile it as well as package shiny and several other dependencies. Although these dependencies are not of concern when $\mathrm{R}$ is run on a personal computer, they can be problematic if the software is running on a server.

GUIProfiler has a convenient characteristic that is missing in the other packages: It connects the profiling tool with an editor to fix the bottlenecks. In the RStudio environment the navigation across the markers pane directly opens the editor on the clicked line of code. If $\mathrm{R}$ is used outside of the RStudio environment, GUIProfiler opens Notepad++ when clicking on the number of the line. This functionality is browser dependent and, at present, it is only implemented for the Internet Explorer 
(and thus the Windows OS) by using ActiveX controls. However, we assume that most of the users will use the RStudio environment where the connection with an external editor is not necessary. Finally we would like to emphasize that GUIProfiler is a useful tool that interactively and graphically helps the users in the difficult task of profiling.

\section{Acknowledgments}

This work was partially supported by the Spanish Minister of Science and Innovation with project "Sanscript" IPT-2012-0093-010000. This funding is gratefully acknowledged.

\section{Bibliography}

W. Chang, J. Cheng, J. J. Allaire, Y. Xie, and J. McPherson. shiny: Web Application Framework for R, 2015. URL https: //CRAN.R-project.org/package=shiny. R package version 0.12.2. [p279]

F. de Villar and A. Rubio. GUIProfiler: Graphical User Interface for Rprof(), 2015. URL https: //CRAN. Rproject. org/package=GUIProfiler. R package version 2.0.1. [p276]

N. Gehlenborg. Nozzle.R1: Nozzle Reports, 2013. URL https://CRAN. R-project.org/package=Nozzle. R1. R package version 1.1-1. [p276]

R. Gentleman, E. Whalen, W. Huber, and S. Falcon. graph: A Package to Handle Graph Data Structures, 2015. R package version 1.48.0. [p279]

K. D. Hansen, J. Gentry, L. Long, R. Gentleman, S. Falcon, F. Hahne, and D. Sarkar. Rgraphviz: Provides Plotting Capabilities for R Graph Objects, 2015. R package version 2.14.0. [p279]

A. A. Klevtsov. proftable: An Alternative to summaryRprof(), 2014. URL https://gi thub.com/noamross/ noamtools/blob/master/R/proftable.R. R function. [p276]

O. Mersmann. microbenchmark: Accurate Timing Functions, 2014. URL https: //CRAN. R-project. org/ package=microbenchmark. R package version 1.4-2. [p276]

D. Schmidt, C. Heckendorf, and W. C. Chen. pbdPAPI: Programming with Big Data-Performance Analysis Tools for R, 2015. URL https://gi thub.com/wrathematics/pbdPAPI. R package. [p276]

The MathWorks Inc. MATLAB - The Language of Technical Computing, Version R2015a. Natick, Massachusetts, 2015. URL http://www. mathworks. com/products/matlab/. [p276]

L. Tierney and R. Jarjour. proftools: Profile Output Processing Tools for R, 2013. URL https: //CRAN . Rproject. org/package=proftools. R package version 0.1-0. [p276]

M. D. Visser, S. M. McMahon, C. Merow, P. M. Dixon, S. Record, and E. Jongejans. Speeding up ecological and evolutionary computations in R; Essentials of high performance computing for biologists. PLoS Computational Biology, 11(3):e1004140, 2015. [p275, 276]

H. Wickham. The split-apply-combine strategy for data analysis. Journal of Statistical Software, 40(1): 1-29, 2011. URL http://www. jstatsoft.org/v40/i01/. [p279]

H. Wickham. lineprof: An Alternative Display for Line Profiling Information, 2014a. R package version 0.1. [p276]

H. Wickham. profr: An Alternative Display for Profiling Information, 2014b. URL https://CRAN.Rproject. org/package=profr. R package version 0.3.1. [p276]

H. Wickham. stringr: Simple, Consistent Wrappers for Common String Operations, 2015. URL https: //CRAN.R-project. org/package=stringr. R package version 1.0.0. [p279]

H. Wickham and W. Chang. devtools: Tools to Make Developing R Packages Easier, 2015. URL https: //CRAN.R-project.org/package=devtools. R package version 1.9.1. [p279]

G. Wilson, D. Aruliah, C. T. Brown, N. P. C. Hong, M. Davis, R. T. Guy, S. H. Haddock, K. Huff, I. M. Mitchell, M. D. Plumbley, et al. Best practices for scientific computing. PLoS Biology, 12(1):e1001745, 2014. [p275]

Y. Xie. knitr: A General-Purpose Package for Dynamic Report Generation in R, 2014. URL https: //CRAN. Rproject.org/package=knitr. R package version 1.6. [p276] 


\author{
Angel Rubio \\ CEIT and TECNUN \\ Universidad de Navarra \\ San Sebastian \\ Spain \\ arubio@ceit.es \\ Fernando de Villar \\ TECNUN \\ Universidad de Navarra \\ San Sebastian \\ Spain \\ fdevillar@gmail.com
}

